\title{
ArcheoSciences
}

Revue d'archéométrie

$31 \mid 2007$

Varia

\section{Les objets de parure en black shales à l'Âge du Fer en Europe celtique : recherche de provenance par l'analyse élémentaire (LA-ICP/MS)}

Ornament artefacts in black shales in the Iron Age in Celtic Europe: research of origin by elementary analysis (LA-ICP/MS)

\section{Anne Baron, Bernard Gratuze et Guirec Querré}

\section{OpenEdition}

Journals

Édition électronique

URL : https://journals.openedition.org/archeosciences/787

DOI : 10.4000/archeosciences.787

ISBN : 978-2-7535-1596-3

ISSN : 2104-3728

Éditeur

Presses universitaires de Rennes

\section{Édition imprimée}

Date de publication : 31 décembre 2007

Pagination : 87-96

ISBN : 978-2-7535-0618-3

ISSN : 1960-1360

Référence électronique

Anne Baron, Bernard Gratuze et Guirec Querré, "Les objets de parure en black shales à l'Âge du Fer en Europe celtique : recherche de provenance par l'analyse élémentaire (LA-ICP/MS) », ArcheoSciences [En ligne], 31 | 2007, mis en ligne le 31 décembre 2009, consulté le 01 février 2022. URL : http:// journals.openedition.org/archeosciences/787 ; DOI : https://doi.org/10.4000/archeosciences.787 


\title{
Les objets de parure en black shales à l'Âge du Fer en Europe celtique : recherche de provenance par l'analyse élémentaire (LA-ICP/MS)
}

\author{
Ornament artefacts in black shales in the Iron Age in Celtic Europe: \\ research of origin by elementary analysis (LA-ICP/MS)
}

\author{
Anne Baron*, Bernard Gratuze** et Guirec Querré***
}

\begin{abstract}
Résumé : Certaines roches sédimentaires, notamment les black shales ont servi à fabriquer des objets de parure pendant l'époque celtique. Ces matériaux sont peu étudiés et les études de caractérisation spécifique peu nombreuses. La présence récurrente de ces objets observés à une large échelle géographique pose plusieurs questions sur la provenance de ces matériaux. Or, la caractérisation de ces derniers constitue la base de toute étude de provenance. La filiation entre les matériaux archéologiques et les sources géologiques demeure essentielle dans la restitution des échanges. L'apport de l'analyse élémentaire par spectrométrie de masse couplée à un plasma inductif avec prélèvement par ablation laser (LA-ICP/MS) permet d'établir des distinctions entre les divers matériaux.

Cette approche permet de dresser un premier bilan. Certains éléments chimiques tels que le tungstène, le molybdène, le lithium, le césium et le rubidium sont discriminants au sein des différents matériaux. Des gisements de différentes régions présentent des caractéristiques chimiques distinctes. Des rapprochements sont faits entre des artefacts archéologiques et des gisements géologiques situés à proximité du site. Cependant, l'attribution des objets archéologiques à des gisements géologiques passe par un meilleur inventaire des sources potentielles.
\end{abstract}

\begin{abstract}
Some sedimentary rocks, especially black shales, were used to make objects such as pendant jewelry during the Celtic period. These materials are not often studied and there are not many specific characteristic studies. The recurrent presence of these artefacts which are studied on a large geographic scale bring up many questions about the origin of these materials. Usually, the origin studies are based on the characterization of materials.

The filiation between archeological artefacts and their geological sources is essential in the restoring of the exchanges. The results of the elementary analysis using laser ablation inductively coupled plasma mass spectroscopy allows to establish distinctions between these different materials.

This approach allows to set up a first conclusion. Some chemical elements such as tungsten, molybdenum, lithium, caesium, and rubidium are discriminants within the different materials. Some outcrops from various regions present different chemical characteristics. Links can be established between archaeological artefacts and geologic deposits situated near the site.

However, the attribution of the archaeological materials to geologic outcrops requires a better inventory of the potential sources.
\end{abstract}

Mots clés : Âge du Fer, Analyses élémentaires, Black shales, Diffusion, Échanges, LA-ICP/MS.

Key words: Black shales, Elementary analysis, Diffusion, Iron Age, LA-ICP/MS, Trade.

*Université de Strasbourg (UMR 7044), UMR 6566 "CREAAH », Laboratoire Archéosciences-Rennes, Bât. 24-25, Université de Rennes 1, Beaulieu, 35042 Rennes cedex. (anne.baron@netcourrier.com)

**IRAMAT, UMR 5060, CNRS/Université d'Orléans, Centre Ernest-Babelon, 3D Rue de la Férollerie, 45071 Orléans cedex 2. (gratuze@cnrs-orleans.fr)

***UMR 6566 "CREAAH ", Laboratoire Archéosciences-Rennes, Bât. 24-25, Université de Rennes 1, Beaulieu, 35042 Rennes cedex. (guirec.querre@ univ-rennes1.fr) 


\section{INTRODUCTION}

La période de l'Âge du Fer (virI $/ \mathrm{I}^{\mathrm{er}}$ siècle av. J.-C.) a donné lieu à de nombreux échanges de matériaux ou d'objets entre les populations celtes installées en Europe. Un type de production tout à fait spécifique prend un essor considérable au cours de cette période et mérite qu'on y porte une attention particulière. Il s'agit d'objets de parure et de récipients fabriqués à partir de diverses roches sédimentaires appelées communément " lignite ».

La distribution de ces objets observée à une large échelle européenne pose plusieurs questions sur la provenance de ces matériaux.

La restitution des filiations entre les matériaux archéologiques employés et les sources géologiques potentielles demeure essentielle dans la restitution des échanges et plus largement dans le système socio-économique qui se développe à l'Âge du Fer. Les aspects de diffusion et de relations avec les centres de production constituent un système commercial spécifique à ces matériaux.

Leur caractérisation constitue donc la base de toute étude de provenance.

\section{Question de terminologie}

La terminologie donnée vis-à-vis de ces matériaux est multiple et complexe.

Ces matériaux sont des roches sédimentaires (à structure feuilletée) de couleur noire à marron constituées d'une fraction minérale et d'une fraction organique (pollens, bois, spores...) en proportions variables. L'identification de ces matériaux fait donc parfois appel à la nomenclature des charbons (Hallsworth et Knox, 1999) mais aussi à celle des roches sédimentaires (Slansky, 1992).

Le manque de caractérisation et la diversité de leur composition a engendré un manque de rigueur dans l'utilisation, parfois trop large et inappropriée du terme "lignite » en archéologie.

$\mathrm{Au} \mathrm{XIX}{ }^{\mathrm{e}}$ siècle, les érudits de l'époque ont utilisé ce terme pour dénommer ces matériaux sans connaître sa constitution. Certains pensaient qu'il s'agissait de bois d'if fossilisé ou de terre agglomérée (Faudel et Bleicher, 1888).

$\mathrm{Au}$ fil du temps, les archéologues ont donc adopté le terme de « lignite " pour qualifier ces objets. Des travaux récents (Teichmüller, 1992; Brechbühl, 2005) ont montré que le lignite au sens géologique n'a pas été employé pour fabriquer ces objets car celui-ci présente un stade de maturation de matière organique assez avancé tel qu'il ne permet pas d'être travaillé par l'homme.
Afin de fixer un terme transitoire plus général dont la définition géologique correspond réellement à la nature géologique des objets, nous proposons le terme de black shales.

Le terme de shale est couramment utilisé par les géologues anglais et offre l'avantage de regrouper plusieurs types de roches : roches carbonatées, carbonées, argilites, schistes bitumineux... Cependant, le jais ne peut être inclus dans cette définition car sa composition est différente. Il est plus riche en carbone, très brillant, à cassure conchoïdale ce qui permet de l'identifier plus aisément.

La traduction de ce terme en français par "schistes noirs » peut porter à confusion car il désigne les schistes au sens large du terme (schistes métamorphiques ou schistes ardoisiers...).

\section{État de la recherche (cf. Fig.1)}

Les travaux publiés en Grande-Bretagne (Allason-Jones et Jones, 1994, 2001; Hunter et al., 1993; Pollard et al., 1981; Watts et Pollard, 1998) sur la caractérisation du Kimmeridge shale et des objets manufacturés en jais par différentes techniques analytiques (FTIR, chromatographie, MEB, fluorescence $\mathrm{X}$ ) ont permis de retracer la diffusion de ces matériaux (à l'échelle insulaire principalement). En Allemagne, différents travaux (Rochna, 1962; Rochna et Mädler, 1984; Grasselt et Volkmann, 1991; Teichmüller, 1992) au début des années 1960 ont permis de poser les premiers jalons de la recherche sur la caractérisation de ces matériaux, mais les études de provenance n'ont pas été établies clairement.

Les études menées récemment en République Tchèque (Valterova, 1998; Venclová, 2001) ont montré que les black shales ont pu être importés ou diffusés sur de longues distances (dans un rayon d'environ $400 \mathrm{~km}$ ). La présence de nombreux ateliers de production et d'une source géologique conséquente située dans le bassin de Kladno près de Prague ont permis de restituer la filiation entre la source d'approvisionnement et les objets finis (cf. Fig. $n^{\circ} 1$ ). Les analyses réalisées (pétrographie et palynologie) ont permis de retracer la diffusion de ce type d'objets pour la période de La Tène moyenne (III ${ }^{\mathrm{e}}-\mathrm{II}^{\mathrm{e}}$ av. J.-C.).

Les travaux antérieurs ont montré que certaines méthodes de caractérisation et notamment la palynologie ne sont pas nécessairement appropriées à l'étude des black shales (Thomas 2003; Baron 2005; Grasselt et Volkmann 1991).

Pour la palynologie, par exemple, une bonne conservation du matériau est nécessaire afin de pouvoir identifier les différents constituants. Les phénomènes d'altération due à sa désagrégation font disparaître de nombreux indices paly- 


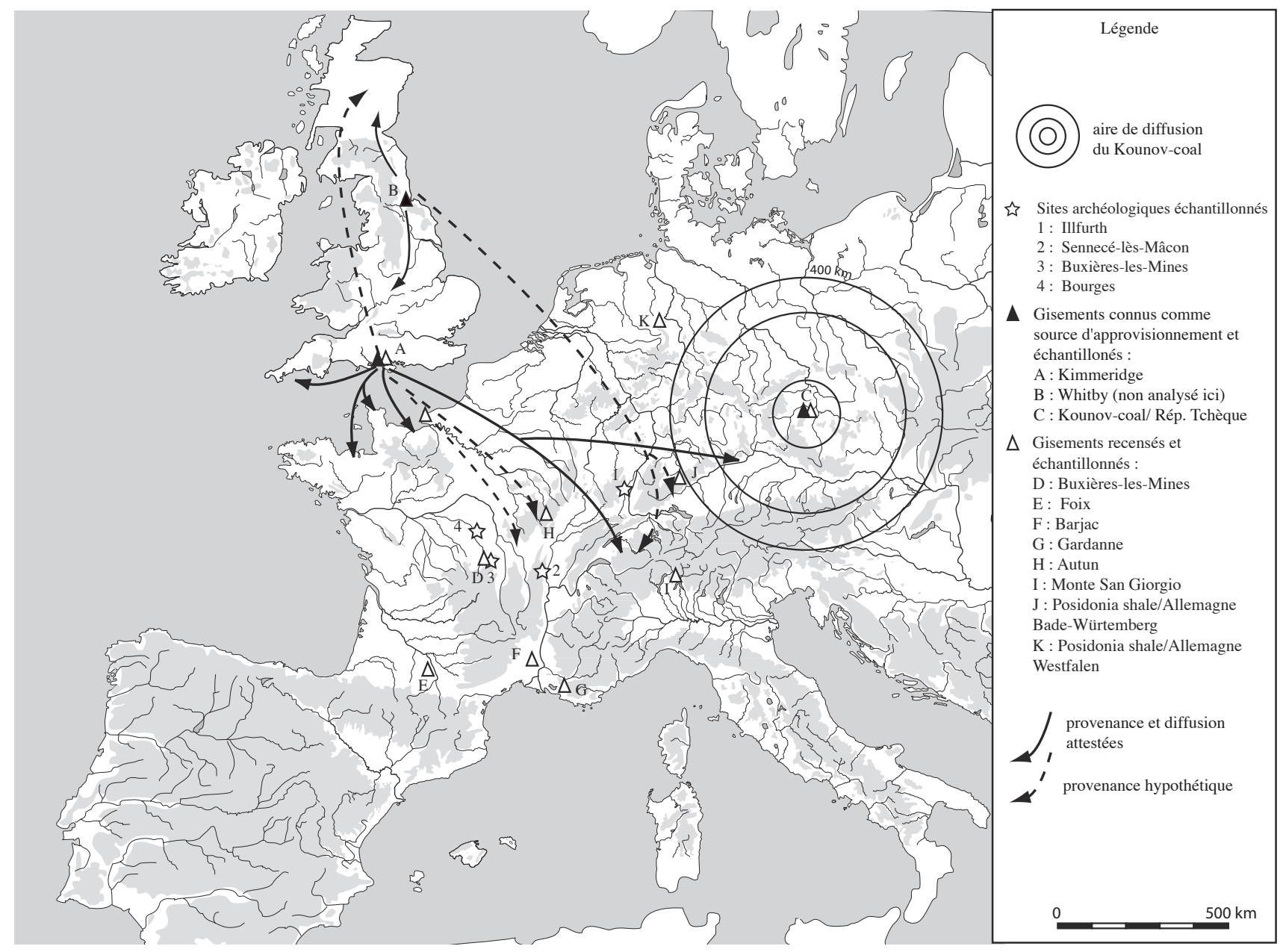

Figure 1 : Carte de diffusion des black shales à l'Âge du Fer en Europe, synthèse d'après les recherches récentes (cartographie A. Baron, fond de carte : Géoatlas()).

Figure 1: Map of diffusion of black shales in the Iron Age in Europe, synthesis according to the recent researches.

nologiques et les chances d'identification sont réduites pour les échantillons archéologiques. Le caractère destructif des méthodes (broyage de l'échantillon) ne permet pas toujours d'élargir l'échantillonnage archéologique.

Certaines études archéologiques ont montré que la diversité des matériaux employés varie d'un site à un autre et peut parfois différer au sein d'une même production artisanale (Eggl, 2000; Venclová, 2001). Les sources géologiques ayant servi à confectionner ces objets peuvent donc être multiples et les matériaux employés avoir des origines diverses.

Afin de résoudre les questions de provenance, notre objectif a été d'élaborer et de tester une méthodologie appropriée permettant une discrimination des sources de matériaux géologiques et archéologiques. Les méthodes utilisées jusqu’à présent telles que la palynologie, la pétrographie, ou l'émission de rayons $\mathrm{X}$ par exemple sont destructrices.
L'analyse chimique élémentaire par le choix de la spectrométrie de masse couplée à un plasma inductif avec prélèvement par ablation laser (LA-ICP/MS) est pertinente dans la mesure où une large gamme d'éléments chimiques peut être mesurée. Cette technique étant peu destructrice, permet d'élargir l'échantillonnage et de multiplier le nombre d'analyses : les échantillons ne nécessitant aucun traitement préalable.

\section{Méthodologie}

La méthode employée se base sur le constat qu'un gisement qui a été exploité à des fins artisanales possède une signature chimique qui lui est propre. Cette signature est conservée dans les objets archéologiques. La question est de 
déterminer les éléments discriminants qui vont permettre de mettre en relation les objets archéologiques avec les sites d'extraction.

La technique LA-ICP/MS a largement été développée et testée dans différentes études portant sur les matériaux du patrimoine culturel (Gratuze et al., 1993; Gratuze et al., 2001 ; Speakman et Neff, 2005; Janssens et Grieken, 2004). Le principe est donc rappelé brièvement ici : l'objet à analyser est placé dans une cellule de quartz. L'ablation se fait grâce à un faisceau laser où la matière prélevée est transportée par un flux d'argon, puis est transmise dans une torche à plasma afin d'être dissociée et ionisée. Les ions sont ensuite dirigés vers des lentilles électrostatiques puis sont focalisés vers le quadripôle qui permet de séparer les éléments selon le rapport $\mathrm{m} / \mathrm{z}$ (masse atomique/charge - avec ici $\mathrm{z}=1$ pour plus de $90 \%$ des éléments). L'utilisation d'étalons géochimiques permet de quantifier les éléments observés. La composition des objets en black shales est proche des roches sédimentaires argileuses. La fraction minérale étant assez importante, les éléments chimiques s'y rapportant ont été dosés. La silice y est l'un des éléments les plus abondants, on peut supposer que sa distribution est l'une des moins hétérogène, elle a donc été choisie comme étalon interne. L'utilisation d'étalons internationaux NIST610 (Pearce et al. 1997) et CORNING B (Brill, 1999; Popelka et al., 2005; Bronk et Freestone, 2001; Verità et al., 1994) permettent de calibrer l'appareil et de vérifier la justesse de la méthode. Faute de disposer d'un étalon certifié pour le carbone, celuici est dosé de façon relative en utilisant une mine de graphite pour laquelle nous avons fixé arbitrairement une teneur de $12 \%$ en graphite.

Le matériau étudié est poreux, il faut donc tenir compte dans l'interprétation des données de la possibilité de modification de la composition des objets durant leur enfouissement : soit par fixation d'éléments provenant du sol (carbonates, phosphates, sulfates, fer, manganèse...), soit par lixiviation ou dissolution par les acides humiques d'éléments ou de composés partiellement solubles.

Les ablations sont réalisées de manière aléatoire sur la surface de l'objet ou sur sa tranche lorsque cela est possible.

Une première série de 2 ablations permet la mesure des éléments majeurs. Celles-ci sont réalisées de manière ponctuelle en trois points de l'échantillon.

Une deuxième série de 2 ablations également permet le dosage des éléments en traces y compris les Terres Rares ( $\mathrm{La}$, $\mathrm{Ce}, \mathrm{Pr}, \mathrm{Tb}$ et $\mathrm{Lu}$ ).

Ayant conscience de l'hétérogénéité du matériau, nous avons fait le choix d'analyser un plus grand nombre d'échantillons par gisement, de façon à raisonner sur une population plus importante. Les teneurs brutes étant difficilement spécifiques à chaque gisement (cas de l'obsidienne par exemple), nous porterons une attention particulière sur la présence ou l'absence de combinaisons d'éléments chimiques susceptibles de caractériser un gisement.

\section{Échantillonnage (cf. Tableau 1 et Fig. 1)}

Malgré l'aspect non-destructif de la méthode, certaines institutions ne souhaitent pas que l'objet soit étudié par des moyens analytiques, ce qui a restreint dans un premier temps le choix des sites et a limité notre échantillonnage.

L'échantillonnage a été orienté vers les sites d'artisanat et certains sites d'habitats en essayant de couvrir au mieux l'aire géographique et les périodes de l'Âge du Fer (Hallstatt et La Tène) en Europe.

D'autres types d'objets ont également été sélectionnés, il s'agit de récipients ou de vases.

Nous constituons actuellement une "lithothèque " de black shales la plus représentative possible tout en connaissant les limites que cela impose (sources épuisées, non connues etc.).

Cet échantillonnage en cours de réalisation constitue une première base sur ce type de matériaux.

Le recensement des échantillons géologiques s'effectue soit par le biais d'institutions ou d'associations géologiques, soit par des prospections géologiques sur le secteur étudié. Cellesci ont été effectuées dans le secteur de Buxières-les-Mines, ainsi que pour les shales à Posidonies par l'intermédiaire des géologues des Landesamt für Geologie d'Allemagne. Le prélèvement d'échantillons géologiques à différents niveaux stratigraphiques voire au sein d'un même niveau a pour objectif d'évaluer l'hétérogénéité des gisements et leur variabilité chimique. Pour les autres gisements échantillonnés, il n'est pas possible d'évaluer cette hétérogénéité à partir des quelques échantillons fournis.

Vingt et un échantillons géologiques et sept échantillons archéologiques ont donc été analysés par LA-ICP/MS dans le cadre de cette étude préliminaire (cf. Tableau 1).

\section{Résultats}

Les éléments majeurs présentant une grande variabilité sont le carbone (entre 1 et $60 \%$ environ), l'oxyde de calcium (entre 1 et $50 \%$ environ), la silice (entre 1 et $60 \%$ environ), l'alumine (entre 5 et $30 \%$ environ) et l'oxyde de fer (entre 2 et $20 \%$ environ) (cf. Fig. 2). Les oxydes de magnésium $(\mathrm{MgO})$, de titane $\left(\mathrm{TiO}_{2}\right)$, de potassium $\left(\mathrm{K}_{2} \mathrm{O}\right)$ 


\begin{tabular}{|c|c|c|c|c|c|}
\hline $\begin{array}{c}\text { Types } \\
\text { échantillons }\end{array}$ & Pays & Région & Localisation & $\begin{array}{l}\text { Description du } \\
\text { matériau/objets }\end{array}$ & Âge/Datation \\
\hline \multicolumn{6}{|l|}{ géologiques } \\
\hline G-OHM-L 018 & Allemagne & Bade-Würtemberg & Ohmden-Lindenhof & Shale à Posidonies & Toarcien \\
\hline G-HOLZ 022 & Allemagne & Bade-Wuirtemberg & Holzmaden & Shale à Posidonies & Toarcien \\
\hline G-OHM 019 & Allemagne & Bade-Wuirtemberg & Ohmden & Shale à Posidonies & Toarcien \\
\hline G-AICH 021 & Allemagne & Bade-Würtemberg & Aichelberg & Shale à Posidonies & Toarcien \\
\hline G-OHM-B 020 & Allemagne & Bade-Würtemberg & Ohmden-Bolzhaiiser & Shale à Posidonies & Toarcien \\
\hline G-BOL 017 & Allemagne & Bade-Würtemberg & Boll & Shale à Posidonies & Toarcien \\
\hline G-WIS 015 & Allemagne & Westfalen & Wistinghausen bei Oerlinghausen & Shale à Posidonies & Toarcien \\
\hline G-MSG 014 & Suisse & Tessin & Monte San Giorgio & dolomitic black shale & Anisien \\
\hline G-MKZ 013 & Rép. Tchèque & Praha & Mšecke Zehrovice & Kounov-coal & Stéphanien \\
\hline G-KIM 012 & Grande-Bretagne & Dorset & Kimmeridge & Shale & Kimméridgien \\
\hline G-AUT 001 & France & Bourgogne & Autun & Schiste bitumineux & Permien \\
\hline G-AUT 002 & France & Bourgogne & Autun & Schiste bitumineux & Permien \\
\hline G-BUX 011 & France & Auvergne & Buxières-les-Mines & Schiste bitumineux & Carbonifère/Permien \\
\hline G-BUX 010 & France & Auvergne & Buxières-les-Mines & Schiste bitumineux & Carbonifère/Permien \\
\hline G-BUX 008 & France & Auvergne & Buxières-les-Mines & Schiste bitumineux & Carbonifère/Permien \\
\hline G-BUX 007 & France & Auvergne & Buxières-les-Mines & Schiste bitumineux & Carbonifère/Permien \\
\hline G-BUX 006 & France & Auvergne & Buxières-les-Mines & Schiste bitumineux & Carbonifère/Permien \\
\hline G-BUX 009 & France & Auvergne & Buxières-les-Mines & Schiste bitumineux & Carbonifère/Permien \\
\hline G-BAJ 005 & France & Languedoc-Roussillon & Barjac & black shale & Éocène \\
\hline G-FOI 004 & France & Midi-Pyrénées & Foix & jais & ind. \\
\hline G-GDN 003 & France & $\begin{array}{c}\text { Provence-Alpes-Côtes } \\
\text { d'Azur }\end{array}$ & Gardanne & lignite & Sénonien \\
\hline \multicolumn{6}{|l|}{ archéologiques } \\
\hline A-BTZ 126 & France & Alsace & Illfurth & frag. de bracelet & Vème s. av. J.-C. \\
\hline A-SNC 096 & France & Bourgogne & Sennecé-lès-Mâcon & frag. de récipient & Ier s. av. J.-C. \\
\hline A-BUX 140 & France & Auvergne & Buxières-les-Mines & déchet de taille & Ilème/Ier s. av. J.-C. \\
\hline A-BUX 139 & France & Auvergne & Buxières-les-Mines & frag. d'ébauche & IIème/Ier s. av. J.-C. \\
\hline A-BUX 138 & France & Auvergne & Buxières-les-Mines & frag. d'ébauche & Ilème/Ier s. av. J.-C. \\
\hline A-BRG 005 & France & Centre & Bourges & frag. de bracelet & VIème/Vème av. J.-C. \\
\hline A-BRG 004 & France & Centre & Bourges & frag. de bracelet & VIème/Vème av. J.-C. \\
\hline
\end{tabular}

Tableau 1 : Liste des échantillons géologiques et archéologiques analysés par LA-ICP/MS.

Table 1: List of geologic and archaeological samples analysed by LA-ICP/MS.

et de phosphore $\left(\mathrm{P}_{2} \mathrm{O}_{5}\right)$ présentent des teneurs homogènes. L'oxyde de manganèse $(\mathrm{MnO})$ et l'oxyde de sodium $\left(\mathrm{Na}_{2} \mathrm{O}\right)$ varient peu et ont des teneurs inférieures à $1 \%$.

Pour les éléments traces (cf. Fig. 2), le strontium et le baryum présentent une grande variabilité ( $\mathrm{Sr}$ : entre 70 et 2500 ppm environ; $\mathrm{Ba}$ : entre 100 et 2600 ppm environ). Les autres éléments traces dosés présentent des variabilités différentes non négligeables. Seuls, le béryllium, le niobium, le terbium et le lutétium varient peu. Leurs teneurs sont relativement homogènes au sein de l'échantillonnage.

Nous présentons ici, seulement certains éléments qui semblent jouer un rôle discriminant dans la caractérisation des sources potentielles; les autres éléments dosés demandant à être mieux étudiés par l'analyse d'autres échantillons. Le faible nombre d'échantillons géologiques analysés ne permet pas actuellement de connaître la variabilité de certaines teneurs au sein d'un gisement. Il est donc difficile de pouvoir déterminer leur pouvoir discriminant.

D'une manière générale, les teneurs mesurées dans les échantillons géologiques sont plus ou moins cohérentes avec les teneurs des échantillons archéologiques. Cependant, ces derniers présentent une hétérogénéité marquée.
Au niveau des éléments majeurs (cf. Tab.2), les échantillons géologiques des shales à Posidonies d'Allemagne s'individualisent nettement par des teneurs en alumine $\left(\mathrm{Al}_{2} \mathrm{O}_{3}\right)$ d'environ $8 \%$, entre 20 et $30 \%$ en silice $\left(\mathrm{SiO}_{2}\right)$ et environ 40-50\% en oxyde de calcium $(\mathrm{CaO})$. Leur composition est relativement homogène exceptée pour l'échantillon provenant d'Allemagne du Nord. Ce dernier (G-WIS 015) présente une teneur plus élevée en $\mathrm{Al}_{2} \mathrm{O}_{3}(19,2 \%)$, plus de $50 \%$ en $\mathrm{SiO}_{2}$ et moins de $15 \%$ en $\mathrm{CaO}$. Il n'est pas rattachable aux autres échantillons allemands.

Les échantillons de Buxières-les-Mines présentent également des compositions assez homogènes (sauf G-BUX 008 et $\mathrm{G}-\mathrm{BUX} 010$ ). Les teneurs en $\mathrm{Al}_{2} \mathrm{O}_{3}$ sont comprises entre 10 et $20 \%$. Les valeurs en $\mathrm{SiO}_{2}$ sont élevées et se situent aux environs de 50-60\%. Par contre, les teneurs en $\mathrm{CaO}$ sont très variables et montre que les couches de ce gisement se sont enrichies de manière sporadique.

La représentation graphique des teneurs en éléments majeurs (cf. Fig. 3) ne permet pas de distinguer des groupes dont la composition élémentaire se définie clairement.

Les échantillons géologiques provenant d'Autun présentent des teneurs d'environ $30 \%$ en $\mathrm{Al}_{2} \mathrm{O}_{3}$, de fortes teneurs 

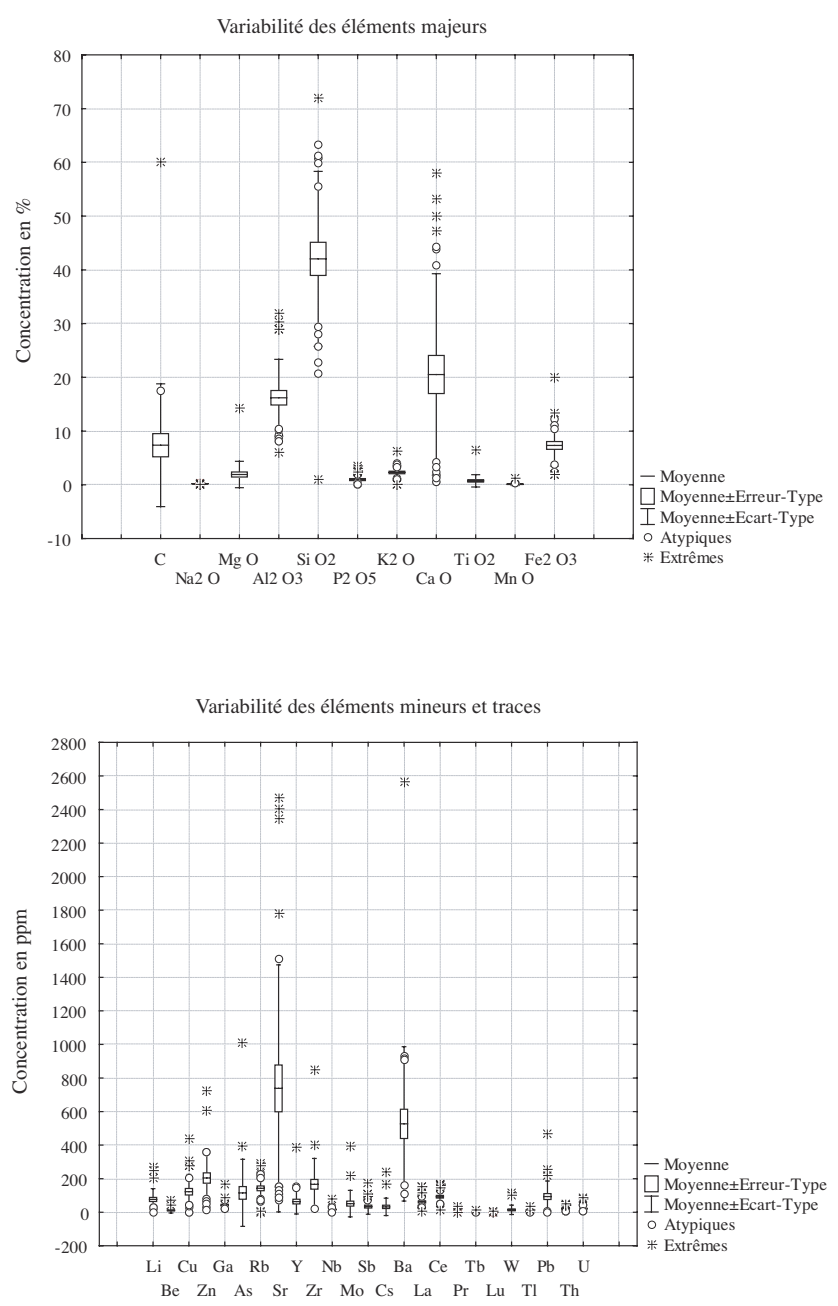

Figure 2 : Variabilités des éléments majeurs, mineurs et traces analysés par LA-ICP/MS.

Figure 2: Variabilities of the major, minor and traces elements analysed by $L A-I C P / M S$.

en $\mathrm{SiO}_{2}$ (plus de $60 \%$ ), mais de très faibles teneurs en $\mathrm{CaO}$ (moins de $1 \%$ ), ce qui ne permet pas de les individualiser des échantillons de Buxières-les-Mines.

Bien que l'échantillon de Foix (G-FOI 004) présente une composition élémentaire particulière, les autres échantillons géologiques de Barjac (G-BAJ 005), de Gardanne (G-GDN 003), de Suisse (G-MSG 014), de République Tchèque (GMKZ 013) et d'Angleterre (G-KIM 033) ne peuvent supporter une interprétation fiable actuellement. En effet, un seul spécimen ne permet pas de caractériser un gisement.

La distinction des matériaux par les teneurs en éléments majeurs reste difficile à appréhender.

Les teneurs des éléments en traces permettent de mieux discriminer les sources (cf. Tab. 3).

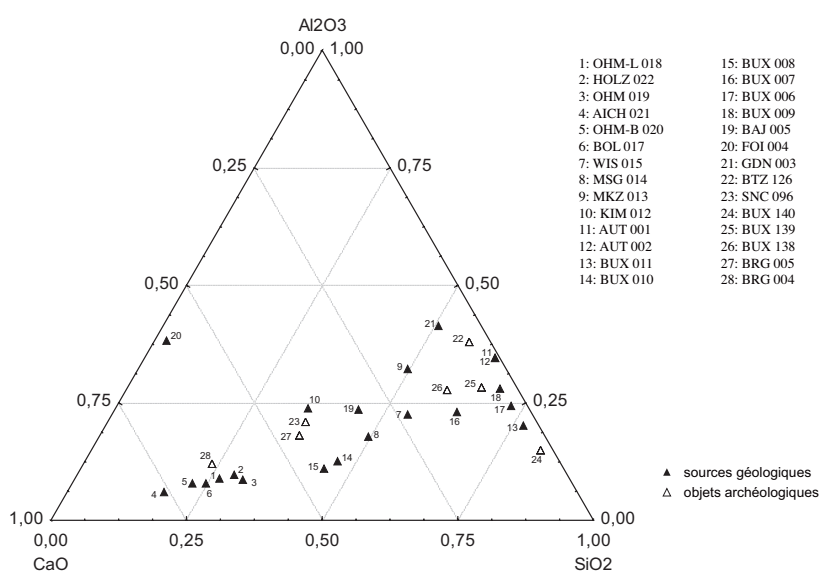

Figure 3 : Diagramme ternaire silice-aluminium-calcium (teneurs exprimées en \%). Représentation des sources géologiques et des objets de parure en black shales en Europe celtique.

Figure 3: Ternary diagram silicium-aluminium-calcium (contents in $\%)$. Representation of geologic outcrops and ornament objects in black shales in celtic Europe.

En effet, la variabilité de certains éléments (cf. Fig. 2) et leur représentation graphique permet d'individualiser plus précisément certains gisements (cf. Fig. 4). Les échantillons d'Autun, notamment, présentent une composition élémentaire caractérisée par de très fortes teneurs en césium et en rubidium (plus de $150 \mathrm{ppm}$ ), mais de très faibles teneurs en molybdène. Ils s'individualisent clairement par rapport aux autres gisements.

Les échantillons de Buxières-les-Mines présentent des teneurs en césium et en molybdène plus disparates (Cs : entre 20 et $60 \mathrm{ppm}$ environ; Mo : entre 10 et $30 \mathrm{ppm}$ environ), mais des teneurs en rubidium élevées $(\mathrm{Rb}$ : entre 150-250 $\mathrm{ppm}$ environ). Deux sous-groupes au sein de ce gisement semblent se définir en ce qui concerne les teneurs en césium : un premier dont les teneurs sont d'environ 20-30 ppm et un deuxième présentant des teneurs supérieures à $50 \mathrm{ppm}$.

Les shales à Posidonies sont plus difficiles à discerner; leurs faibles teneurs en césium (moins de $5 \mathrm{ppm}$ ), en rubidium (60-80 ppm), mais élevées en molybdène (40-60 ppm) ne permet pas de les individualiser des autres gisements. Cependant, l'échantillon d'Allemagne du Nord se distingue toujours par sa composition élémentaire particulière, semblant se rapprocher de celle des schistes bitumineux de Buxières-les-Mines.

D'autres gisements analysés se particularisent également par leur composition : l'échantillon de jais de Foix (G-FOI 004) est caractérisé par de très faibles teneurs en rubidium, césium et molybdène, et l'échantillon G-KIM 012 s'identifie par une très forte teneur en molybdène, mais faible en césium (10 ppm). 


\begin{tabular}{|c|c|c|c|c|c|c|c|c|c|c|c|}
\hline $\begin{array}{c}\text { Types } \\
\text { échantillons } \\
\end{array}$ & $\mathrm{C}$ & $\mathrm{Na}_{2} \mathrm{O}$ & $\mathrm{Mg} \mathrm{O}$ & $\mathrm{Al}_{2} \mathrm{O}_{3}$ & $\mathrm{SiO}_{2}$ & $\mathrm{P}_{2} \mathrm{O}_{3}$ & $\mathrm{~K}_{2} \mathrm{O}$ & $\mathrm{CaO}$ & $\mathrm{TiO}_{2}$ & Mn O & $\mathrm{Fe}_{2} \mathrm{O}_{3}$ \\
\hline \multicolumn{12}{|l|}{ géologiques } \\
\hline G-OHM-L 018 & 2,84 & 0,31 & 2,25 & 8,80 & 29,80 & 0,26 & 1,72 & 47,20 & 0,36 & 0,15 & 6,02 \\
\hline G-HOLZ 022 & 2,38 & 0,30 & 2,50 & 9,34 & 31,50 & 0,19 & 1,84 & 43,80 & 0,36 & 0,15 & 7,36 \\
\hline G-OHM 019 & 1,57 & 0,24 & 1,86 & 8,64 & 34,90 & 0,32 & 1,65 & 44,20 & 0,35 & $<0,10$ & 6,03 \\
\hline G-AICH 021 & 2,37 & 0,25 & 1,96 & 6,08 & 20,80 & 3,51 & 1,28 & 58 & 0,27 & 0,18 & 4,96 \\
\hline G-OHM-B 020 & 2,55 & 0,23 & 2,13 & 8,07 & 25,70 & 0,35 & 1,52 & 53,20 & 0,30 & 0,17 & 5,36 \\
\hline G-BOL 017 & 2,24 & 0,26 & 2,26 & 8,02 & 28,10 & 0,17 & 1,64 & 50,10 & 0,39 & 0,15 & 6,38 \\
\hline G-WIS 015 & 1,51 & 0,29 & 1,51 & 19,20 & 52,60 & 0,47 & 2,65 & 14,60 & 0,88 & 0,11 & 6 \\
\hline G-MSG 014 & 0,98 & 0,27 & 14,2 & 13 & 40,90 & 0,17 & 6,20 & 17,60 & 0,36 & $<0,10$ & 6,15 \\
\hline G-MKZ 013 & 17,4 & 0,29 & 0,64 & 17,10 & 29,90 & 2,35 & 3,88 & 7,12 & 0,55 & $<0,10$ & 20 \\
\hline G-KIM 012 & 13,5 & 0,32 & 1,21 & 18,60 & 31,50 & 1,02 & 2,30 & 23,60 & 0,58 & $<0,10$ & 7,25 \\
\hline G-AUT 001 & 2,46 & 0,10 & 0,81 & 28,90 & 60,80 & 0,23 & 3,22 & 0,55 & 0,98 & $<0,10$ & 1,87 \\
\hline G-AUT 002 & 2,46 & 0,10 & 0,81 & 28,90 & 60,80 & 0,23 & 3,22 & 0,55 & 0,98 & $<0,10$ & 1,87 \\
\hline G-BUX 011 & 3,46 & 0,15 & 1,05 & 14,60 & 63,20 & 1,22 & 2,12 & 1,51 & 0,43 & $<0,10$ & 12,20 \\
\hline G-BUX 010 & 1,76 & 0,16 & 1,45 & 11,70 & 48,70 & 0,86 & 1,90 & 27,90 & 0,38 & 0,39 & 4,63 \\
\hline G-BUX 008 & 1,68 & 0,15 & 1,53 & 10,10 & 47,20 & 0,64 & 1,84 & 30,50 & 0,36 & 0,33 & 5,57 \\
\hline G-BUX 007 & 4,46 & 0,20 & 1,75 & 17,20 & 53,80 & 3,05 & 2,40 & 7,61 & 0,81 & 0,15 & 8,56 \\
\hline G-BUX 006 & 12,10 & 0,15 & 0,93 & 18,10 & 61,30 & 0,17 & 2,38 & 1,65 & 0,72 & $<0,10$ & 2,48 \\
\hline G-BUX 009 & 1,61 & 0,14 & 1,48 & 21,60 & 59,90 & 0,94 & 3,39 & 1,89 & 0,68 & $<0,10$ & 8,26 \\
\hline G-BAJ 005 & 12,30 & 0,13 & 0,97 & 18,10 & 39 & 0,18 & 1,07 & 18 & 0,53 & 0,11 & 9,53 \\
\hline G-FOI 004 & 60,2 & 0,31 & 1,80 & 14,30 & 0,96 & 0,10 & 0,17 & 16,50 & $<0,10$ & $<0,10$ & 5,18 \\
\hline G-GDN 003 & 5,12 & 0,17 & 2 & 30,30 & 42,10 & 0,45 & $<0,10$ & 4,32 & 6,47 & 0,12 & 8,74 \\
\hline \multicolumn{12}{|l|}{ archéologiques } \\
\hline A-BTZ 126 & 0,79 & $<0,10$ & 1,05 & 32 & 55,6 & 0,84 & 2,68 & 2,40 & 0,70 & $<0,10$ & 3,69 \\
\hline A-SNC 096 & 15,80 & 0,23 & 1,18 & 14,80 & 29,30 & 2,59 & 1,92 & 22,3 & 0,48 & $<0,10$ & 11,20 \\
\hline A-BUX 140 & 4,12 & 0,19 & 1,03 & 11,60 & 72,10 & 0,91 & 2,47 & 1,24 & 0,47 & 1,21 & 4,61 \\
\hline A-BUX 139 & 7,47 & 0,30 & 1,56 & 19,40 & 50,80 & 1,34 & 3,72 & 3,35 & 0,79 & $<0,10$ & 10,40 \\
\hline A-BUX 138 & 6,24 & 0,27 & 1,72 & 20,40 & 49,30 & 2,01 & 3,40 & 7,06 & 0,73 & $<0,10$ & 8,75 \\
\hline A-BRG 005 & 5,54 & 0,20 & 1,32 & 14,60 & 34 & 0,53 & 2,45 & 27,20 & 0,49 & 0,10 & 13,40 \\
\hline A-BRG 004 & 11,60 & 0,19 & 0,89 & 10,30 & 22,80 & 2,40 & 1,47 & 40,8 & 0,33 & $<0,10$ & 8,97 \\
\hline
\end{tabular}

Tableau 2 : Teneurs en éléments majeurs (exprimées en \%).

Table: Contents in major elements (in \%).

Les autres matériaux analysés, dont les échantillons archéologiques de Bourges, d'Illfurth et de Sennecé-lès-Mâcon, ne permettent pas de définir des groupes précis par manque d'analyses sur un même matériau.

D'autres élément traces, notamment le lithium et le tungstène permettent de confirmer la séparation de certains groupes (cf. Fig. 5).

Les shales à Posidonies d'Allemagne sont caractérisés par des teneurs en lithium assez faibles (entre 20 et $30 \mathrm{ppm}$ ), des teneurs assez fortes en molybdène (entre 50 et $60 \mathrm{ppm}$ ), mais des teneurs extrêmement faibles en tungstène. La composition chimique de l'échantillon d'Allemagne du Nord (G-WIS 015) diffère et est difficilement attribuable à un groupe.
Le groupe d'échantillons de Buxières-les-Mines est homogène. Il est caractérisé par des teneurs en lithium entre 60 et $100 \mathrm{ppm}$ environ, des teneurs assez faibles en molybdène comprises entre 10-30 ppm environ. Enfin, ces schistes bitumineux s'identifient par de fortes teneurs en tungstène (entre 10-20 ppm).

Les schistes bitumineux d'Autun se différencient nettement par une très forte teneur en lithium et en tungstène (plus de 200 ppm) et de très faibles quantités en molybdène (moins de 3 ppm).

L'échantillon de jais provenant de Foix (G-FOI 004) se distingue également par une teneur en tungstène de $20 \mathrm{ppm}$, mais de très faibles quantités en lithium et en molybdène.

Les autres échantillons analysés présentent des teneurs encore trop disparates pour les différencier. 


\begin{tabular}{|c|c|c|c|c|c|c|c|c|c|c|c|c|c|c|c|c|c|c|c|c|c|c|c|c|c|}
\hline $\begin{array}{c}\text { Types } \\
\text { échantillons } \\
\end{array}$ & $\mathbf{L i}$ & Be & $\mathrm{Cu}$ & $\mathbf{Z n}$ & $\mathbf{G a}$ & As & $\mathbf{R b}$ & $\mathrm{Sr}$ & $\mathbf{Y}$ & $\mathbf{Z r}$ & $\mathbf{N b}$ & Mo & Sb & Cs & $\mathbf{B a}$ & $\mathbf{L a}$ & $\mathrm{Ce}$ & Pr & $\mathbf{T b}$ & Lu & $\mathbf{w}$ & TI & $\mathbf{P b}$ & Th & $\mathbf{U}$ \\
\hline \multicolumn{26}{|l|}{ géologiques } \\
\hline G-OHM-L 018 & 28 & 2 & 201 & 358 & 26 & 22 & 79 & 2401 & 113 & 173 & 8 & 66 & 3 & 4 & 384 & 75 & 165 & 24 & 3 & 1 & 1 & 15 & 92 & 14 & 16 \\
\hline G-HOLZ 022 & 32 & 2 & 191 & 273 & 25 & 25 & 89 & 2469 & 51 & 126 & 8 & 51 & 3 & 5 & 298 & 51 & 93 & 11 & 1 & 1 & 1 & 16 & 84 & 7 & 12 \\
\hline G-OHM 019 & 26 & 2 & 84 & 234 & 22 & 21 & 88 & 1039 & 30 & 61 & 7 & 59 & 1 & 5 & 309 & 32 & 75 & 9 & 1 & 0 & 1 & 10 & 26 & 8 & 9 \\
\hline G-AICH 021 & 20 & 1 & 153 & 206 & 17 & 19 & 61 & 1248 & 36 & 81 & 5 & 47 & 3 & 3 & 163 & 33 & 64 & 8 & 1 & 0 & 0 & 12 & 68 & 4 & 7 \\
\hline G-OHM-B 020 & 25 & 2 & 150 & 206 & 18 & 19 & 67 & 1509 & 55 & 100 & 7 & 47 & 2 & 4 & 192 & 44 & 81 & 11 & 1 & 1 & 0 & 12 & 61 & 5 & 9 \\
\hline G-BOL 017 & 26 & 2 & 188 & 359 & 22 & 24 & 72 & 1781 & 48 & 131 & 8 & 35 & 3 & 4 & 269 & 42 & 79 & 10 & 1 & 1 & 1 & 9 & 75 & 5 & 9 \\
\hline G-WIS 015 & 68 & 3 & 79 & 196 & 52 & 34 & 165 & 655 & 43 & 161 & 18 & 27 & 1 & 11 & 930 & 62 & 119 & 14 & 1 & 1 & 2 & 6 & 52 & 17 & 7 \\
\hline G-MSG 014 & 42 & 3 & 100 & 109 & 22 & 144 & 115 & 150 & 29 & 405 & 17 & 216 & 47 & 13 & 226 & 28 & 51 & 5 & 1 & 1 & 2 & 33 & 140 & 37 & 39 \\
\hline G-MKZ 013 & 36 & 75 & 128 & 609 & 44 & 1013 & 86 & 2347 & 83 & 140 & 12 & 50 & 112 & 12 & 420 & 60 & 76 & 9 & 1 & 1 & 2 & 5 & 469 & 28 & 14 \\
\hline G-KIM 012 & 101 & 3 & 169 & 80 & 36 & 29 & 139 & 453 & 71 & 118 & 12 & 392 & 1 & 10 & 655 & 81 & 103 & 14 & 2 & 1 & 1 & 9 & 47 & 12 & 20 \\
\hline G-AUT 001 & 205 & 9 & 46 & 85 & 50 & 16 & 236 & 153 & 38 & 201 & 33 & 3 & 20 & 165 & 404 & 100 & 168 & 21 & 1 & 1 & 116 & 6 & 83 & 39 & 15 \\
\hline G-AUT 002 & 268 & 9 & 42 & 82 & 51 & 16 & 293 & 151 & 48 & 232 & 31 & 2 & 19 & 237 & 378 & 92 & 158 & 19 & 2 & 1 & 103 & 6 & 85 & 41 & 16 \\
\hline G-BUX 011 & 77 & 21 & 70 & 157 & 41 & 245 & 229 & 95 & 18 & 125 & 16 & 15 & 91 & 56 & 438 & 37 & 43 & 5 & 0 & 0 & 12 & 2 & 50 & 45 & 27 \\
\hline G-BUX 010 & 61 & 3 & 48 & 96 & 27 & 155 & 150 & 1192 & 42 & 268 & 9 & 18 & 31 & 21 & 400 & 61 & 117 & 14 & 2 & 1 & 10 & 1 & 29 & 25 & 32 \\
\hline G-BUX 008 & 57 & 2 & 29 & 65 & 24 & 92 & 143 & 611 & 14 & 55 & 9 & 30 & 32 & 23 & 324 & 27 & 50 & 6 & 1 & 0 & 10 & 2 & 40 & 10 & 18 \\
\hline G-BUX 007 & 75 & 15 & 48 & 87 & 35 & 98 & 205 & 130 & 16 & 167 & 21 & 19 & 55 & 34 & 431 & 42 & 65 & 8 & 1 & 0 & 10 & 3 & 30 & 22 & 41 \\
\hline G-BUX 006 & 61 & 44 & 2 & 26 & 30 & 19 & 186 & 72 & 42 & 156 & 14 & 9 & 174 & 51 & 293 & 47 & 63 & 8 & 1 & 1 & 16 & 1 & 3 & 17 & 6 \\
\hline G-BUX 009 & 121 & 10 & 63 & 52 & 41 & 396 & 275 & 111 & 25 & 119 & 23 & 16 & 57 & 66 & 393 & 50 & 72 & 8 & 1 & 0 & 23 & 3 & 61 & 37 & 22 \\
\hline G-BAJ 005 & 74 & 9 & 71 & 202 & 25 & 87 & 113 & 637 & 19 & 165 & 16 & 79 & 4 & 24 & 222 & 39 & 49 & 6 & 1 & 0 & 5 & 2 & 40 & 16 & 80 \\
\hline G-FOI 004 & 1 & 11 & 2 & 13 & 35 & 0 & 2 & 457 & 155 & 18 & 2 & 1 & 1 & 0 & 106 & 7 & 13 & 2 & 3 & 2 & 21 & 0 & 0 & 5 & 5 \\
\hline G-GDN 003 & 47 & 13 & 275 & 727 & 166 & 2 & 5 & 339 & 387 & 852 & 78 & 4 & 3 & 1 & 686 & 156 & 162 & 38 & 11 & 4 & 1 & 1 & 114 & 19 & 3 \\
\hline \multicolumn{26}{|l|}{ archéologiques } \\
\hline A-BTZ 126 & 248 & 15 & 33 & 162 & 69 & 28 & 196 & 442 & 26 & 65 & 25 & 4 & 1 & 35 & 524 & 59 & 119 & 14 & 1 & 0 & 17 & 2 & 97 & 49 & 89 \\
\hline A-SNC 096 & 84 & 4 & 277 & 193 & 43 & 52 & 119 & 519 & 148 & 169 & 10 & 87 & 2 & 8 & 913 & 156 & 147 & 21 & 3 & 1 & 2 & 3 & 106 & 14 & 25 \\
\hline A-BUX 140 & 79 & 6 & 85 & 299 & 51 & 169 & 215 & 86 & 35 & 64 & 14 & 28 & 75 & 27 & 826 & 54 & 101 & 11 & 1 & 0 & 20 & 4 & 69 & 19 & 21 \\
\hline A-BUX 139 & 70 & 9 & 41 & 201 & 88 & 211 & 225 & 355 & 34 & 169 & 19 & 32 & 97 & 29 & 2562 & 63 & 72 & 9 & 1 & 0 & 9 & 2 & 133 & 21 & 11 \\
\hline A-BUX 138 & 74 & 16 & 108 & 111 & 44 & 228 & 204 & 524 & 18 & 136 & 17 & 30 & 106 & 31 & 515 & 51 & 67 & 7 & 1 & 0 & 9 & 2 & 220 & 19 & 15 \\
\hline A-BRG 005 & 75 & 5 & 436 & 236 & 42 & 36 & 138 & 267 & 44 & 100 & 15 & 17 & 2 & 10 & 906 & 60 & 87 & 14 & 1 & 0 & 1 & 1 & 256 & 13 & 7 \\
\hline A-BRG 004 & 56 & 4 & 303 & 288 & 30 & 31 & 93 & 475 & 108 & 101 & 8 & 51 & 3 & 8 & 578 & 113 & 130 & 20 & 2 & 1 & 1 & 5 & 84 & 9 & 15 \\
\hline
\end{tabular}

Tableau 3 : Teneurs en éléments mineurs et traces (exprimées en ppm).

Table 3: Contents in minor and traces elements (in ppm).

Si pour les échantillons géologiques analysés, il est possible de distinguer certains groupes, cela est plus difficile pour les matériaux archéologiques. En effet, leurs teneurs très variées ne permettent pas toujours d'établir des corrélations avec les gisements potentiels. De plus, la composition de certains échantillons provenant d'un même site ne présente pas toujours des teneurs similaires (cf. teneurs élémentaires pour le site de Bourges Tableaux 2 et 3).

Cependant, les échantillons provenant du site archéologique de Buxières-les-Mines présentent des teneurs plus ou moins proches de celles des échantillons géologiques provenant du même secteur (cf. Fig. 4 et 5). Le test de plusieurs échantillons à différents endroits de la couche a permis d'observer la variabilité de la signature chimique du gisement et de pouvoir les comparer avec les échantillons retrouvés sur le site archéologique. Les objets découverts à Buxières-lesMines ont été fabriqués à partir des schistes bitumineux de l'Aumance situés à proximité.
Les autres objets archéologiques provenant de Bourges, Illfurth ou Sennecé-lès-Mâcon sont difficilement rattachables à l'un des gisements analysés. Malgré le faible nombre d'analyses effectuées sur du mobilier archéologique (d'autres analyses sont actuellement en cours), les matériaux employés sont nettement différents de ceux des sources d'approvisionnement potentielles recensées à ce jour (exceptés pour le site de Buxières-les-Mines).

Les éléments discriminants dans l'état actuel de l'avancement des travaux sont le lithium, le molybdène, le césium, le tungstène et le rubidium.

D'une manière générale, il semble que les alcalins jouent un rôle important dans la composition chimique de ces roches.

Par ailleurs, le lithium semble être un excellent traceur pour ces matériaux, ainsi que le tungstène dont la présence pourrait s'expliquer par l'héritage sédimentaire provenant de minéraux lourds. 


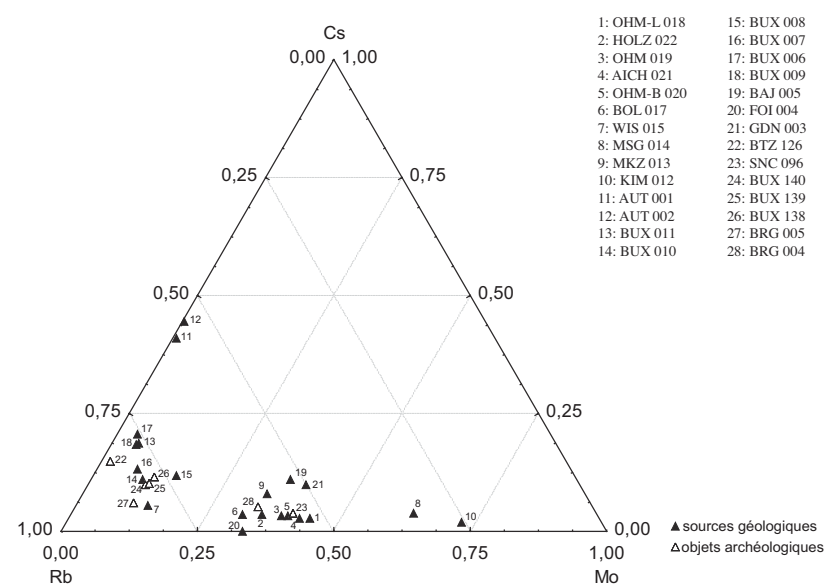

Figure 4: Diagramme ternaire rubidium-molybdène-césium. Représentation des sources géologiques et des objets de parure en black shales en Europe celtique.

Figure 4: Ternary diagram rubidium-molybdenum-cesium. representation of geologic outcrops and ornament objects in black shales in Celtic Europe.

Le thorium, le strontium, le baryum et ou bien encore le zirconium sont des éléments dont le pouvoir discriminant semble prometteur.

Les terres rares (particulièrement le niobium, le terbium et le lutétium), généralement bons traceurs géologiques, ne semblent pas pour ces matériaux être utilisables comme éléments discriminants.

\section{Conclusions et Perspectives}

Les mesures de composition élémentaires par LA-ICP/MS permettent de différencier les origines de certains des objets de parure en black shales.

D'autres analyses (en cours de réalisation) seront nécessaires pour établir les potentialités de cette approche. L'étude du pouvoir discriminant d'éléments comme le zirconium, le cuivre, le baryum, l'uranium, le cérium, ou le strontium par exemple, reste à développer.

Cette approche a permis de mettre en évidence la variété des teneurs élémentaires présentes dans les matériaux employés et la complexité à déterminer leurs provenances. Cette diversité ne permet pas dans l'état actuel de la recherche de définir clairement un type de roche précis. Il peut s'agir de schiste bitumineux, d'argilites, voire de pélites ou toutes autres roches sédimentaires d'origine détritique dont les modes d'enfouissement peuvent différer et avoir subi des transformations différentes. La détermination des minéraux présents dans ces roches est nécessaire pour affiner cette
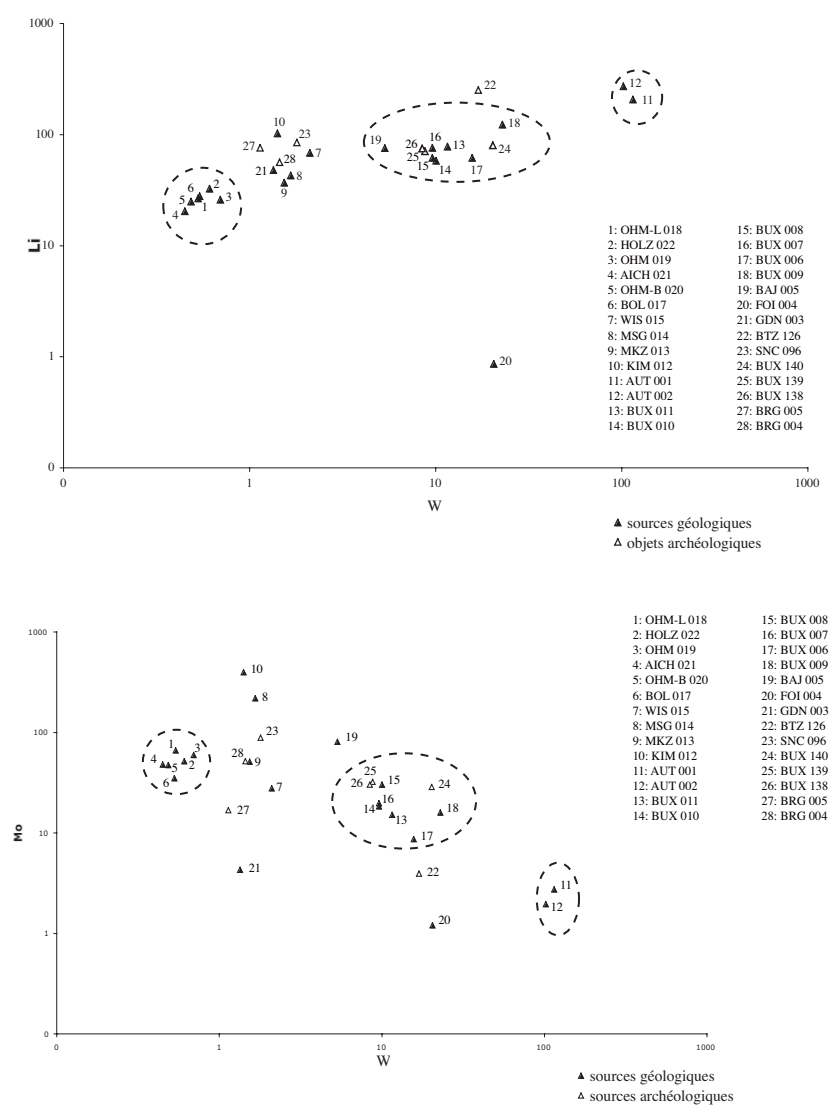

Figure 5 : Diagrammes binaires tungstène-lithium et tungstènemolybdène (teneurs exprimées en ppm). Échelle logarithmique. Représentation des objets de parure en black shales et de leurs sources potentielles en Europe celtique.

Figure 5: Binary diagrams tungsten-lithium and tungsten-molybdenum (contents in ppm). Logarithmic scale. Representation of archaeological artefacts and their potentials outcrops in Celtic Europe.

caractérisation. La poursuite de cette recherche s'organisera donc en couplant d'autres méthodes analytiques, et notamment la diffraction $\mathrm{X}$, avec les compositions élémentaires obtenues par LA-ICP/MS.

\section{Bibliographie}

Allason-Jones (L.), Jones (D.-M.), 1994. Museum Notes, Archaeologia Aeliana, 5e série, vol XXII, The Society of Antiquaries of NewCastle-Upon-Tyne, tiré à part, p. 263-272.

Allason-Jones (L.) et Jones (D.-M.), 2001. Identification of jet artefacts by reflected light microscopy, European Journal of archaeology, vol. 4, 2, London, p. 233-251. 
BARON (A.), 2005. Origine et provenance des objets en "lignite" à l'Âge du Fer: essai de détermination, mémoire de DEA, Université de Strasbourg, 2 vol.

BRECHBÜHL (S.), 2005. Untersuchung verschiedener Konservierungsmethoden auf erdfeuchtem bis nassem archäologischen Gagat und anderen Kaustobiolithen, Mémoire de fin d'étude, Haute école d'arts appliqués Arc, Filière conservation-restauration, orientation archéologie, éthnographie, Bern, Suisse.

Brill (R.-H.), 1999. Chemical analyses of early glasses, New York, The Corning Museum of Glass, 2 vol.

Bronk (H.), et Freestone (I. C.), 2001. A Quasi Non-destructive Microsampling Technique for the Analysis of Intact Glass Objects By Sem/edxa, Archaeometry, 43(4), p. 517-527.

EGGL (Ch.), 2000. Sapropelitfunde aus dem Oppidum von Manching, Hausarbeit zur Erlangung des Magister Artium an der LudwigMaximilians-Universität München, sous la direction de Dr. A. Lang et Dr. V. Bierbrauer, septembre 2000, Insitut für Vor- und Frühgeschichte und Provinzialrömische Archäologie.

Faudel et Bleicher, 1888. Contribution à l'étude de la céramique préromaine et des matières premières usitées par les populations anciennes de l'Alsace, de la Lorraine, du Nord de l'Afrique, Matériaux pour une étude préhistorique de l'Alsace, Colmar, p. 99-112.

Grasselt (Th.), et Volkmann (N.), 1991. Latènezeitlicher Ringschmuck aus Thüringen, Alt-Jahresschrift des Museums für Ur-und Frügeschichte Thüringens, 26, p. 179-196.

Gratuze (B.), Giovagnoli (A.), Barrandon (J.-N.), Telouk (Ph.) et Imbert (J.-L.) 1993. Apport de la méthode ICP-MS couplée à l'ablation laser pour la caractérisation des archéomatériaux, Revue d'Archéométrie, Rennes, 17, p. 89-104.

Gratuze (B.), Blet-Lemarquand (M.) et Barrandon (J.-N.), 2001. Mass spectrometry with laser sampling: A new tool to characterize archaeological material, Journal of Radionanalytical and Nuclear Chemistry, 247-3, p. 645-656.

Hallsworth (C. R.) et KnOx (R. W.) 1999. Classification of sediments and sedimentary rocks, BGS Rock Classification Scheme, Volume 3, British Geological Survey Research Report, RR 9903, NERC, Nottingham, 1999.

Hunter (F. J.), McDonnel (J. G.), Pollard (A. M), Morris (C. R.) et Rowlands (C. C.), 1993. The scientific identification of archaeological jet-like artefacts, Archaeometry, 35, 1, Blackwell publishing for University of Oxford, p. 69-89.

Janssens (K.), Van Grieken (R.), 2004. Non-destructive Microanalysis of Cultural Heritage Materials, (Eds.), Comprehensive Analytical Chemistry, vol. XLII, Elsevier, Amsterdam, the Netherlands, 800 p.

Pearce (N. J. G.), Perkins (W. T.), Westgate (J. A.), Gorton (M. T.), Jackson (S. E.), Neal (C. R.) et Chenery (S. P.), 1997. A compilation of new and published major and trace element data for NIST SRM 610 and SRM 612 glass reference materials, Geostandards Newsletter, XXI, p. 114-115.

Pollard (A. M.), Bussel (G. D.) et Baird (D. C.), 1981. The analytical investigation of Early Bronze Age jet and jet-like material from the Devizes Museum, Archaeometry, 23, 2, Blackwell publishing for University of Oxford, p. 139-167.

Popelka (R. S.), Glascock (M. D.), Robertshaw (P.) et Wood (M.), 2005. Laser Ablation ICP-MS of African Glass Trade Beads, in Speakman (R. J.) et H. Neff (eds), Laser Ablation ICP-MS in Archaeological Research, Albuquerque, University of New Mexico Press, p. 84-93.

Rochna (O.), 1962. Hallstattzeitlicher Lignit-und Gagat-Schmuck Zur Verbreitung, Zeitstellung und Herkunft, Fundberichte aus Schwaben, Neue Folge 16, Stuttgart, 44-83.

Rochna (O.) et Mädler (K.), 1984. Das Tonschierfer-, Sapropelit-, Gagat- und Wasmaterial der Heuneburg, in Sievers (S.) (dir.), Die Kleinfunde der Heuneburg, Die Funde aus den Grabfunden von 1950-1979, Heuneburgstudien V, Römischgermanische Forschungen, 42, Mainz, 91-94, taf. 18-26.

SLANSKy (M.) 1992. Terminologie et classification des roches sédimentaires formées de silice, silicates, carbonates et phosphates, Manuels et Méthodes, n 22, Orléans, Éditions du BRGM.

Speakman (R. J.) et NefF (H.), 2005. Laser Ablation-ICP-MS in archaeological research, Albuquerque, University of New Mexico Press, 200 p.

Teichmüller (M.), 1992. Organic petrology in the service of archaelogy, International Journal of Coal Archaeology, 20, Elsevier Science Publishers B. V., Amsterdam, p. 1-21.

Thomas (S.), 2003. Étude pétrographique et palynologique de mobilier en "lignite" protohistorique. Caractérisation et étude de provenance, Mémoire de DESS, Université de Bourgogne, Dijon.

Valterova (P.), 1998. Palynology of the Kounov-type sapropelite, in Venclova (dir.) Mšecké Zehrovice in Bohemia, Archaeological background to a celtic hero $3^{\text {rd }}-2^{\text {nd }}$ cent. B.-C., Sceaux, Kronos édition, coll. "Chronothèque; 2 ", p. 311.

Venclova (N.) 2001. Vyroba a sidla v Dobe Laténské, Projekt Lodenice (Production and settlement in the La Tène period, the Lodenice project), Prague, Institut d'Archéologie de Prague.

Verità (M.), Basso (R.), Wypyski (M. T.) et Koestler (R. J.), 1994. X-ray Microanalysis of Ancient Glassy Materials: A Comparative Study of Wavelength Dispersive and Energy Dispersive Techniques, Archaeometry, 36(2), p. 241-251.

Watts (S.) et Pollard (A. M.), 1998. Identifying archaeological jet and jet-like artifacts using FTIR, Résumé des communications du colloque Infrared User's Group (IRUG) septembre 1995, p. 37-52. 